\title{
Preface: HINPw6 - Hellenic Institute of Nuclear Physics; 6th International workshop; Perspectives on Nuclear Physics; from fundamentals to applications
}

\author{
Athena Pakou ${ }^{1}$, Dennis Bonatsos ${ }^{2}$, Georgios A.Lalazissis ${ }^{3}$ and George A. Souliotis ${ }^{4}$ \\ ${ }^{1}$ Department of Physics and HINP, University of Ioannina, 45110 Ioannina, Greece \\ ${ }^{2}$ Institute of Nuclear and Particle Physics, National Centre for Scientific Research "Demokritos", GR-15310 Aghia \\ Paraskevi, Attiki, Greece \\ ${ }^{3}$ Department of Physics, Aristotle University of Thessaloniki, Thessaloniki GR-54124, Greece \\ ${ }^{4}$ Department of Chemistry, National and Kapodistrian University of Athens and HINP, 15771 Athens, Greece
}

The sixth International workshp - HINPw6 of the Hellenic Institute of Nuclear Physics (HINP) was held on May 14 to 16, 2021, by means of a zoom platform, due to Covid-19 restrictions. HINP is a virtual Institute of nonprofitable character that constitutes a form of collaboration between three Nuclear Physics Laboratories, the University of Ioannina, the Aristotle University of Thessaloniki, and the National \& Kapodistrian University of Athens. Members of the National Centre for Scientific Research "Demokritos" are also participating. Details on the Institute can be found in http://hinp.physics.uoi.gr/

One of the main goals of HINP is the collaboration between Nuclear Physics Laboratories in Greece with strong international links, promoting exchange of ideas among senior researchers, postdocs and graduate students with experimental and theoretical background. The HINP series of workshops are devoted to this goal.

The present web-based conference was coorganized by the National and Kapodistrian University of Athens (NKUA) and the University of Ioannina (UoI). While this is the $6^{\text {th }}$ workshop of HINP, it is the third one with a clearly international character, following HINPw4, held at the U. of Ioannina in May 2017, and HINPw5, organized at the Aristotle
U. of Thessaloniki in April 2019, on which further information can be found at http://hinp.physics.uoi.gr/Workshops.htm

Over 70 participants attended the workshop, welcomed by the Dean of the School of Natural Sciences of NKUA, Professor Ioannis Emmanouil, and the Director of the Physical Chemistry Laboratory of NKUA, Professor Andreas Koutselos. We warmly thank both of them. The workshop included scientific talks by 25 invited speakers from several parts of the world, including Europe, USA, and China. In total 47 speakers reported their research on various topics in

specific sessions of

1. Nuclear Structure

2. Advances in Nuclear Facilities

3. Nuclear Reactions with weakly bound nuclei

4. Nuclear Astrophysics

5. Heavy-Ion Reactions and Rare Isotope Production

6. Applications

Posters were replaced by 5 min flash talks, a novelty highly appreciated by the participants.

While most of the talks were focused on fundamental open problems, an effort has been made by the organizing committee to demonstrate the unified character of 
fundamental and applied research. Thus the opening talk, given by Nicolas Alamanos from CEA Paris-Saclay, was on "Nuclear Physics; The Societal Impact - A project for Greece?" Highlights in the same direction were the talks by Sherry Yennello from Texas A\&M on "Advancing research in Texas through Experiments in Medical Isotope Science", and by Luis Acosta from UNAM on "The AMS technique as an important tool for the measurement of astrophysical cross sections", as well as a flash talk by Vasileios Soukeras from INFN-LNS, Catania on "Low energy proton induced reactions for application purposes".

Important new results were outlined in various subfields. We warmly thank all speakers for their high level talks. We especially thank our invited speakers with intriguing subjects on Nuclear Structure (relativistic Brueckner-Hartree-Fock and relativistic Energy Density Functional theories for finite nuclei; chirality and wobbling phenomena; octupole deformation in the "nuclear clock" metastable state in $\left.{ }^{229} \mathrm{Th}\right)$, Nuclear Astrophysics $\left({ }^{12} \mathrm{C}+{ }^{12} \mathrm{C}\right.$ subbarrier fusion; ternary fission in low density matter; astrophysics at the n-TOF facility) and Nuclear Reactions from light weakly bound halo nuclei $\left({ }^{6,8} \mathrm{He},{ }^{11} \mathrm{Be},{ }^{15} \mathrm{C}\right)$ to heavy neutron rich drip line elements and the connection of the nuclear equation of state to the interplay between fusion and quasi-fission processes in low-energy nuclear reactions. We further warmly thank our invited speakers presenting special advances in their laboratories for weak interactions, including the TwinSol facility at the Notre Dame U., the IEAP at the Czech Technical U. in Prague, and the NUMEN project - MAGNEX facility in the INFN-LNS, Catania.

Once more we feel that we would like to warmly thank all our colleagues and collaborators from abroad, and especially those, who constantly support with their presence and their inspiring talks our workshops: Nicolas Alamanos from CEA Paris-Saclay, Francesco Cappuzzello from INFN-LNS and the U. of Catania, and Peter Ring from the Technical U. of Munich. 